\title{
Ga-induced atom wire formation and passivation of stepped $\mathrm{Si}(112)$
}

\author{
P. C. Snijders* and S. Rogge \\ Kavli Institute of NanoScience Delft, Delft University of Technology, 2628 CJ Delft, The Netherlands \\ C. González, R. Pérez, J. Ortega, and F. Flores \\ Facultad de Ciencias, Departamento de Física Teórica de la Materia Condensada, Universidad Autónoma de Madrid, \\ Madrid 28049, Spain \\ H. H. Weitering \\ Department of Physics and Astronomy, The University of Tennessee, Knoxville, Tennessee 37996, USA \\ and Condensed Matter Sciences Division, Oak Ridge National Laboratory, Oak Ridge, Tennessee 37831, USA
}

(Received 13 May 2005; published 29 September 2005)

\begin{abstract}
We present an in-depth analysis of the atomic and electronic structure of the quasi-one-dimensional (1D) surface reconstruction of $\mathrm{Ga}$ on $\mathrm{Si}(112)$ based on scanning tunneling microscopy and spectroscopy (STM and STS), Rutherford-backscattering spectrometry (RBS), and density functional theory (DFT) calculations. A new structural model of the $\mathrm{Si}(112) 6 \times 1-\mathrm{Ga}$ surface is inferred. It consists of Ga zigzag chains that are intersected by quasiperiodic vacancy lines or misfit dislocations. The experimentally observed meandering of the vacancy lines is caused by the coexistence of competing $6 \times 1$ and $5 \times 1$ unit cells and by the orientational disorder of symmetry breaking $\mathrm{Si}-\mathrm{Ga}$ dimers inside the vacancy lines. The $\mathrm{Ga}$ atoms are fully coordinated, and the surface is chemically passivated. STS data reveal a semiconducting surface and show excellent agreement with calculated local density of states (LDOS) and STS curves. The energy gain obtained by fully passivating the surface calls the idea of step-edge decoration as a viable growth method toward 1D metallic structures into question.
\end{abstract}

DOI: $10.1103 /$ PhysRevB.72.125343

PACS number(s): 68.35.-p, 68.37.Ef, 73.20.At, 81.07.Vb

\section{INTRODUCTION}

Nature only provides a few one-dimensional (1D) electronic systems, such as carbon nanotubes, ${ }^{1}$ organic charge transfer salts, and inorganic blue bronzes (see, for example, the discussion in Ref. 2). Electrons confined to one dimension are fundamentally different from the quasiparticles of Fermi-liquid theory. ${ }^{3}$ In 1D, even in the case of arbitrary low interaction strength, the single-particle description of the system breaks down and must be replaced by a description based on collective excitations. ${ }^{3}$ Experimental realization and verification of this Luttinger-liquid phenomenon continues to capture the imagination of physicists, especially since the fabrication of structurally uniform 1D nanostructures now appears to be within the realm of possibilities.

A very intuitive approach to produce $1 \mathrm{D}$ systems is to utilize high-index silicon surfaces. ${ }^{4}$ Based on the concept of metal-adatom step-edge decoration, deposition of a submonolayer amount of metal atoms onto a stepped $\mathrm{Si}$ surface is expected to result in a single domain of quasi-1D, metallic atomic wires, i.e., an atom wire array. ${ }^{4}$ In contrast to, for example, carbon nanotubes, such a single-domain-surface quantum wire array would be easily accessible to both nanoscopic and macroscopic techniques such as scanning tunneling microscopy and spectroscopy (STM and STS), photoemission spectroscopy, and (surface) transport measurements. In addition, the coupling strength between the atom wires can be tuned by changing the miscut angle of the vicinal $\mathrm{Si}$ surface, i.e., adjusting the separation between the wires. ${ }^{5}$

Indeed such single-domain 1D metallic systems have been produced on high-index Si surfaces. ${ }^{5-9}$ However, the metal adatoms in these studies are generally not adsorbed at the step edges. Instead, rather complicated reconstructions are formed with chains of metal atoms that are incorporated into the (111)-like terraces in the unit cell., $5,10,11$ This questions the idea of forming atom wires via step-edge decoration on Si. Furthermore, it was noticed ${ }^{12}$ that these $1 \mathrm{D}$ atomicscale systems all exhibit intrinsic spatial disorder in the atomic structure, which will have important consequences for electronic transport in these systems. It should be noted that in the case that step-edge decoration does not occur in these studies, the miscut or vicinal orientation of the Si surfaces mainly serves to create a single domain surface reconstruction; similar or "parent" reconstructions exist on the corresponding planar surfaces. These single-domain quantum wire arrays have been studied successfully with angleresolved photoelectron spectroscopy (ARPES) $)^{5,9,13-15}$ and transport measurements. ${ }^{16}$

In this paper, we investigate the formation of Ga chains on the vicinal $\mathrm{Si}(112)$ surface. A structural model for this interface was devised by Jung, Kaplan, and Prokes (the JKP model). ${ }^{17-22}$ The unit cell of the bulk-terminated vicinal $\mathrm{Si}(112)$ surface contains a double-width (111)-like terrace with single (111)-like steps. Based on low-energy electron diffraction (LEED) and Auger-electron spectroscopy (AES) experiments, ${ }^{17-19}$ it was proposed that $\mathrm{Ga}$ atoms adsorb at the step edges of the bulk-terminated unit cell, thus forming atom rows along the [1 $\overline{1} 0]$ direction. Missing Ga atoms or vacancies in these Ga rows align into quasi-1D vacancy lines that run orthogonal to the Ga rows, resulting in the observed $6 \times 1$ periodicity. In this model, the Ga coverage is $\frac{1}{6}$ of a 

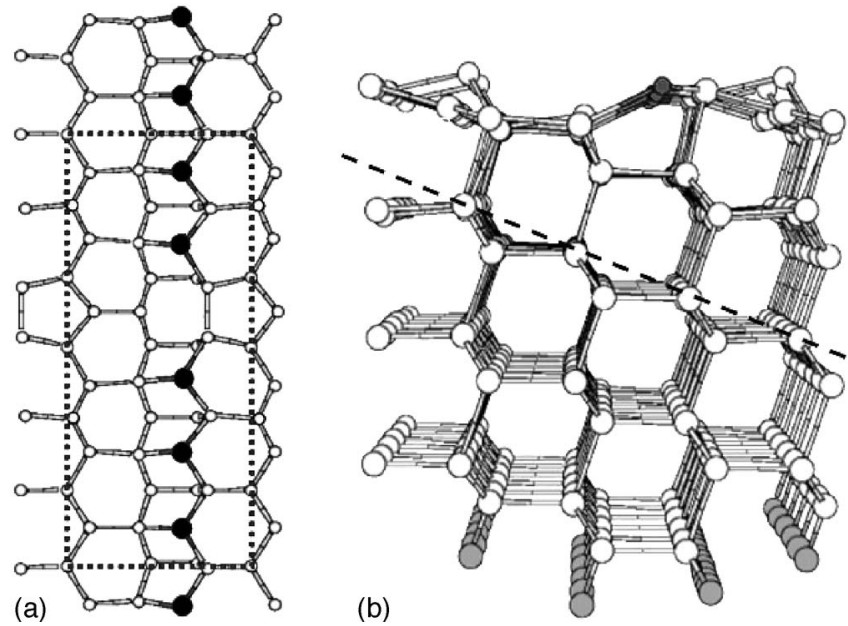

FIG. 1. (a) Top view and (b) side view of the JKP model of the $\mathrm{Si}(112) 6 \times 1-\mathrm{Ga}$ surface. In (a) the $6 \times 1$ unit cell is indicated by the dotted lines. In (b) a (111) plane is indicated. Si atoms: light; Ga atoms: dark.

Si(111) bilayer, or five atoms per $6 \times 1$ unit cell. ${ }^{18}$ Later STM experiments by Baski et al. ${ }^{20,23}$ seemed to confirm this model. These authors observed a well-ordered array of single-atom rows with a regular row spacing $9.4 \AA$, equal to the step-edge spacing of bulk terminated $\mathrm{Si}(112)$; see for example Fig. 1 of Ref. 20. A side-view representation and a top-view representation of this JKP model is shown in Fig. 1.

As a consequence of the threefold coordination of the adsorption sites, the trivalent Ga atoms are fully coordinated. There are no partially filled dangling bonds on the $\mathrm{Ga}$ atoms and the covalently bonded $\mathrm{Ga}$ atoms would not contribute any state density near the Fermi level. However, an interesting feature which has remained largely unnoticed in literature is the fact that within the JKP model, there should exist a metallic dangling bond wire that is located on the row of $\mathrm{Si}$ surface atoms located in between the Ga rows. However, the predicted 1D metallicity turned out to be unstable with respect to a Jahn-Teller distortion, leaving only one unpaired electron per $6 \times 1$ unit cell. Interestingly, the resulting electronic structure implied the existence of conduction channels orthogonal to the Ga chains. ${ }^{22}$

We have performed a detailed scanning tunneling microscopy study of the $\mathrm{Si}(112) 6 \times 1-\mathrm{Ga}$ surface. Because of the unprecedented resolution in the STM images of the $\mathrm{Si}(112) 6 \times 1-\mathrm{Ga}$ surface, a detailed investigation of the atomic structure of the $\mathrm{Si}(112) 6 \times 1-\mathrm{Ga}$ surface could be carried out. Extensive density functional theory calculations have been performed to explore new candidate structural models. Theoretical STM images were calculated for the new structures and compared with the experimental STM images. From a detailed analysis of all experimental and theoretical information, a new structural model for the Si(112)6 $\times 1-G a$ surface emerged, which shows excellent agreement with the experimental evidence. It contains two $\mathrm{Ga}$ atom rows amounting to a total of ten $\mathrm{Ga}$ atoms per 6 $\times 1$ unit cell, consistent with Rutherford-backscattering spectroscopy (RBS) experiments. The two Ga rows form zigzag chains while quasiperiodic vacancy lines intersect these
Ga chains. The observed meandering of the vacancy lines can also be fully explained within this model. STS measurements show that the surface is semiconducting; these measurements are consistent with our band structure calculations and theoretical STS simulations. This paper presents a follow-up of an initial report ${ }^{12}$ with new data and provides a more detailed and in-depth analysis, including a detailed comparison between spatially resolved STS and theoretical local density of states (LDOS) calculations.

\section{EXPERIMENTAL AND THEORETICAL PROCEDURES}

Experiments were carried out in an ultra high vacuum system with a base pressure $<5 \times 10^{-11}$ mbar. The system was equipped with a Ga-effusion cell, direct current sample heating facilities, an Omicron variable temperature STM, and a LEED system. An $n$-type $\operatorname{Si}(112)$ wafer $[\sim 5$ $\times 10^{14} \mathrm{~cm}^{-3}$, orientation $\pm 2^{\circ}$ of the nominal (112) orientation] was cut into $(10 \times 2)-\mathrm{mm}^{2}$ samples and rinsed in acetone and isopropanol. After introduction into UHV, the samples were degassed at $775 \mathrm{~K}$ overnight and subsequently the sample temperature was slowly raised to $1025 \mathrm{~K}$ and kept there for $4 \mathrm{~h}$. Next, the sample was flashed at $1475 \mathrm{~K}$ to remove the native oxide. During resistive heating, the current was directed parallel to the nanofacets of the clean (112) surface (i.e., in the [1 $\overline{1} 0]$ direction) in order to avoid currentinduced step bunching. The surface reconstruction was prepared in two different ways. In the "one-step" procedure, Ga was deposited with the Si substrate held at $825 \pm 50 \mathrm{~K} .{ }^{24} \mathrm{In}$ the "two-step" procedure, Ga is deposited onto a $\mathrm{Si}(112)$ substrate kept at room temperature. After Ga deposition, the sample was annealed at about $825 \pm 50 \mathrm{~K}$ to form the $6 \times 1$ reconstruction and to desorb excess $\mathrm{Ga}$ atoms. ${ }^{20}$ Both surface preparation procedures resulted in identical LEED patterns and STM images. The pressure remained below 2 $\times 10^{-10}$ mbar during sample preparation. The sample temperature during sample preparation was measured using an optical pyrometer. STM and STS experiments were performed at room temperature and at low temperature $(\sim 40$ $\mathrm{K})$ using etched tungsten tips. STM images of the filled and empty electronic states were obtained with a constant current between 0.05 and $0.2 \mathrm{nA}$ and bias voltages between 1 and $2 \mathrm{~V}$. STS data were acquired with a set point of $0.3 \mathrm{nA}$ at $1 \mathrm{~V}$.

RBS experiments were carried out at the AMOLF Institute in Amsterdam to determine the amount of $\mathrm{Ga}$ atoms per surface unit cell. A normal incident $2.0 \mathrm{MeV} \mathrm{He}{ }^{+}$ion beam from a Van de Graaf accelerator was backscattered from the Si crystal and detected at a backscattering angle of $165^{\circ}$. The beam current was typically about $20 \mathrm{nA}$.

The atomic and electronic structure of new candidate structural models for the $\mathrm{Si}(112) 6 \times 1-\mathrm{Ga}$ surface, corresponding to $\mathrm{Ga}$ coverages ranging from 5 to $11 \mathrm{Ga}$ atoms per $6 \times 1$ unit cell were explored using an efficient local-orbital (LO) density functional theory (DFT) technique (the FIREBALL96 code). ${ }^{25}$ In these calculations, we have used a minimal atomiclike basis set using the following cutoff radii $\left(R_{c}\right)$ for the definition of the FIREBALL96 orbitals: ${ }^{26} R_{c}(\mathrm{Si})=5.0$, 
$R_{c}(\mathrm{Ga})=5.2$. For the most promising structures, plane-wave (PW) DFT calculations (CASTEP code) ${ }^{27}$ were also performed to check the validity of the FIREBALL96 findings. In these PW calculations, we have used a kinetic energy cutoff $E_{c}$ of $200 \mathrm{eV}$ for the definition of the PW basis set, and four special $k$ points for the Brillouin zone sampling (test calculations with $250 \mathrm{eV}$ and eight special $k$ points were also performed). In both the LO and PW calculations, we have used a slab of $11 \mathrm{Si}$ layers with hydrogen atoms saturating the bonds of the deeper Si layers (see Fig. 1).

Using the DFT local-orbital Hamiltonian of the surface together with nonequilibrium Keldysh-Green-function techniques, ${ }^{28,29}$ we calculated theoretical STM images for the new relaxed atomic structures. The theoretical images were then compared with the experimental STM images. In our approach, we divide the total Hamiltonian $\hat{H}$ of our tipsample system, $\hat{H}=\hat{H}_{t}+\hat{H}_{s}+\hat{H}_{\text {int }}$ into three parts: $\hat{H}_{t}, \hat{H}_{s}$, and $\hat{H}_{\text {int }}$ referring to the tip, sample, and their interaction. $\hat{H}_{s}$ is obtained from the FIREBALL96 code used to calculate the $\mathrm{Si}(112) 6 \times 1-\mathrm{Ga}$ surface; $\hat{H}_{t}$ is calculated using the same DFT local-orbital code for a $\mathrm{W}$ tip having a pyramid with four atoms, attached to a $\mathrm{W}-(100)$ surface; $\hat{H}_{\text {int }}$ is obtained using a dimer approximation, whereby the different tipsample hopping interactions $\hat{T}_{t s}$ are calculated from the dimer formed by the respective tip and sample atoms whose interaction we want to obtain (it is shown in Ref. 30 that this approximation yields a good description of the STM images if orbitals with long-range tails are used in the hopping calculations). A more detailed description of our procedure to obtain theoretical STM images can be found in Refs. 30 and 31. Making use of the total Hamiltonian and the Keldysh Green-function techniques, we can calculate the tunneling current from the following equation: ${ }^{28}$

$$
\begin{aligned}
I= & \frac{4 \pi e}{\hbar} \int_{-\infty}^{\infty} d \omega \operatorname{Tr}\left[\hat{T}_{t s} \hat{\rho}_{s s}(\omega) \hat{D}_{s s}^{r}(\omega) \hat{T}_{s t} \hat{\rho}_{t t}(\omega) \hat{D}_{t t}^{a}(\omega)\right] \\
& \times\left[f_{t}(\omega)-f_{s}(\omega)\right],
\end{aligned}
$$

where

$$
\hat{D}_{s s}^{r}=\left[\hat{1}-\hat{T}_{s t} \hat{g}_{t t}^{r}(\omega) \hat{T}_{t s} \hat{g}_{s s}^{r}(\omega)\right]^{-1}
$$

and

$$
\hat{D}_{t t}^{a}=\left[\hat{1}-\hat{T}_{t s} \hat{g}_{s s}^{a}(\omega) \hat{T}_{s t} \hat{g}_{t t}^{a}(\omega)\right]^{-1}
$$

include all the interface multiple scattering processes. $\mathrm{Tr}$ stands for the Trace of the current matrix. $\hat{g}_{s s}^{a(r)}$ and $\hat{g}_{t t}^{a(r)}$ are the advanced (retarded) Green functions of the sample and the tip, respectively (calculated taking $\hat{T}_{t s}=0$ ); $\hat{\rho}_{s s}$ and $\hat{\rho}_{t t}$ are the sample and tip density of states (also for $\hat{T}_{t s}=0$ ); and $f_{t}\left(f_{s}\right)$ the Fermi distribution functions.

In the tunneling regime, $\hat{T}_{t s}$ is very small and $\hat{D}_{s s}^{r}$ and $\hat{D}_{t t}^{a}$ can be replaced by $\hat{I}$. In this limit, for zero temperature, we recover the following equation:

$$
I=\frac{4 \pi e}{\hbar} \int_{E_{F}}^{E_{F}+e V} d \omega \operatorname{Tr}\left[\hat{T}_{t s} \hat{\rho}_{s s}(\omega) \hat{T}_{s t} \hat{\rho}_{t t}(\omega)\right],
$$

which we have used to calculate the STM images of the different surface structures.

We should comment that the detailed comparison between theory and experimental results that we intend in this work requires the use of Eq. (4) instead of other simpler approaches (like the Tersoff-Hamann formalism) that are common in the literature. Our method includes a realistic description of the geometry and the full electronic structure of the tip and incorporates, quantitatively, the influence of the tunneling parameters (bias and current conditions) and the tip-sample distance. ${ }^{30,31}$ This quantitative accuracy, crucial to understanding the contradictory experimental results in terms of contrast and symmetry of the STM images of an apparently simple system like $\mathrm{O} / \mathrm{Pd}(111)-2 \times 2$ (Ref. 31 ) is necessary in our case to discriminate among all the different surface structures that have been analyzed in this work. Notice, in particular, that we show below that our proposed model is fully compatible with the STM images by Baski et $a l .{ }^{20}$ provided that their tunneling parameters are used in the simulation of the STM images. On top of these advantages, we have to mention that our approach does not require a significantly larger computational time than other simpler methods, as Eq. (4) provides a very compact procedure for calculating the tip-sample tunneling current that takes full advantage of the LDOS $\left(\hat{\rho}_{s s}\right.$ and $\left.\hat{\rho}_{t t}\right)$ obtained from our DFT calculations.

\section{STM OBSERVATIONS}

The high-index $\operatorname{Si}(112)$ surface is tilted $19.5^{\circ}$ away from the (111) surface toward (001). But the pristine $\mathrm{Si}(112)$ surface is not thermodynamically stable and breaks up into approximately 10-nm-wide nanofacets of reconstructed (111)like and (337)-like planes. ${ }^{23,32}$ An STM image of pristine $\mathrm{Si}(112)$ is shown in Fig. 2(a). One might expect that metal deposition on this surface would result in the formation of metallic nanowires in these prepatterned grooves. However, it was shown by Baski et al. ${ }^{23}$ that upon deposition and postannealing of a submonolayer amount of $\mathrm{Ga}$, the faceted $\mathrm{Si}(112)$ surface undergoes a massive restructuring. It returns to its basal (112) orientation, reconstructing as described in the introduction. This preparation procedure of deposition and postannealing of the surface resulted in a reproducible self-limiting surface reconstruction with a $6 \times 1$ unit cell. ${ }^{18} \mathrm{~A}$ large-scale STM image of Ga-covered $\mathrm{Si}(112)$ is shown in Fig. 2(b). The nanoscale facets have developed into large anisotropic (112) terraces that can extend for up to microns along the $[1 \overline{1} 0]$ direction but are less than $100 \mathrm{~nm}$ wide. On the terraces, the vacancy lines appear as dark trenches, which run perpendicular to the step edges present in this image. Closer inspection reveals that these vacancy lines are not exactly straight, but their position fluctuates around an average position. As discussed below, this is due to the coexistence of $6 \times 1$ and $5 \times 1$ units in the surface and due to the presence of intrinsic fluctuations in the vacancy lines. ${ }^{12}$ 


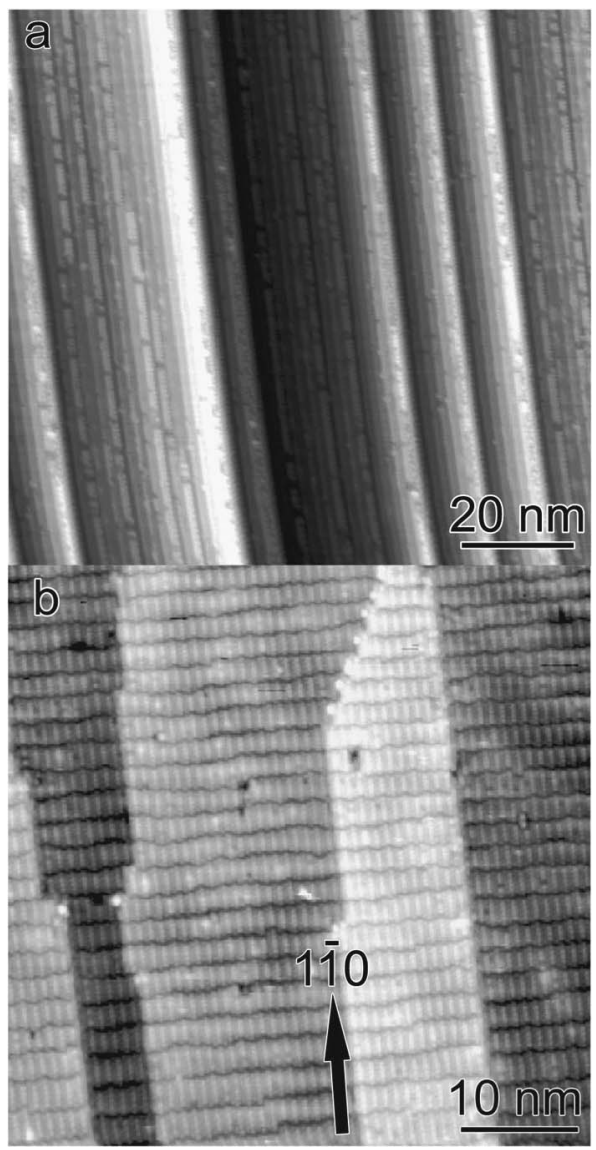

FIG. 2. (a) STM image of pristine $\mathrm{Si}(112)$. (b) STM image of the Ga-covered $\mathrm{Si}(112)$ surface. Tunneling conditions: $1.5 \mathrm{~V}$, $0.1 \mathrm{nA}$ and $2 \mathrm{~V}, 0.1 \mathrm{nA}$ for (a) and (b), respectively.

Detailed atomic resolution STM images were acquired to investigate the atomic structure of the $\mathrm{Si}(112) 6 \times 1-\mathrm{Ga}$ surface. In Fig. 3, an atomic resolution empty-state STM image is shown. Note that this particular area of the surface shows both $5 \times 1$ and $6 \times 1$ unit cells as indicated in the figure. Two parallel atom rows are observed per unit cell, running in the [11̄0] direction, intersected by the quasiperiodical vacancy lines. Comparing this image with the results of Baski et al., ${ }^{20}$ we observe the same spacing of the brightest atom rows (i.e., $9.4 \AA$ ). Furthermore, the mixed periodicities and the similar LEED pattern (see Refs. 20 and 18, respectively) indicate that the same surface reconstruction is studied here. Consequently, we conclude that the brightest atom rows in Fig. 3 are the same atom rows as imaged by Baski et al. ${ }^{20}$ (henceforth, the "step-edge Ga row"). But in addition, we observe a second atom row lying in between the brighter rows. In terms of the JKP model, this row of atoms could be interpreted as the Si dangling bond row, which might form a quasi-1D band. However, these two parallel atomic lines clearly form a zigzag pattern as indicated in Fig. 3 (see also Fig. 13), which results in a structural asymmetry in the vacancy line. This is in contradiction with the JKP model, which implies mirror plane symmetry with respect to the (110) plane in the vacancy line.

In Fig. 4, a set of registry-aligned dual-bias images is presented. These images have been recorded simultaneously

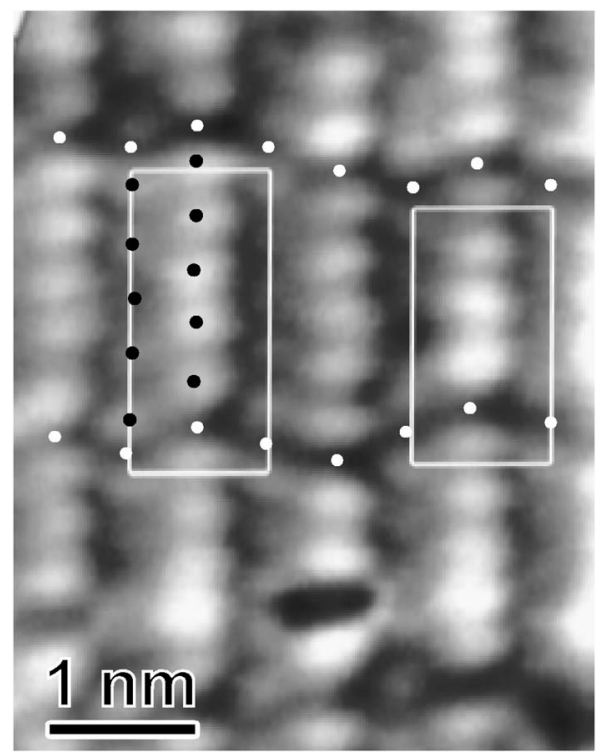

FIG. 3. Empty-state STM image of the $\operatorname{Si}(112) n \times 1-G a$ surface. In this particular surface area, both $5 \times 1$ and $6 \times 1$ unit cells are present, as indicated. Atomic positions are indicated with black dots. The position of the vacancies in both atomic rows is indicated with white dots. Tunneling conditions: $1.5 \mathrm{~V}, 0.2 \mathrm{nA}$.

on the same area of the surface, but with opposite tunneling bias polarities resulting in a set of spatially correlated emptystate and filled-state images. In this case, the empty-state image, Fig. 4(a), has suffered from a slight decrease in resolution, as compared with Fig. 3, but the asymmetry in the vacancy line is still visible. In the filled-state image, Fig. 4(b), a relatively big, symmetric protrusion prevents a detailed observation of the atomic structure in the vacancy line. As in the empty-state image, two parallel atom rows are also visible in the filled-state image. They form a ladder structure instead of the zigzag pattern of the rows observed in the empty-state image.

RBS measurements were performed to determine experimentally the amount of $\mathrm{Ga}$ at the surface. Integration of the Ga peak in the backscattered He spectrum yielded an amount of $9 \pm 1 \mathrm{Ga}$ atoms per $6 \times 1$ unit cell, to be compared with five Ga per $6 \times 1$ unit cell for the JKP model of Fig. 1 .

In summary, these experimental results (STM and RBS) consistently show that the step-edge decorated JKP model of

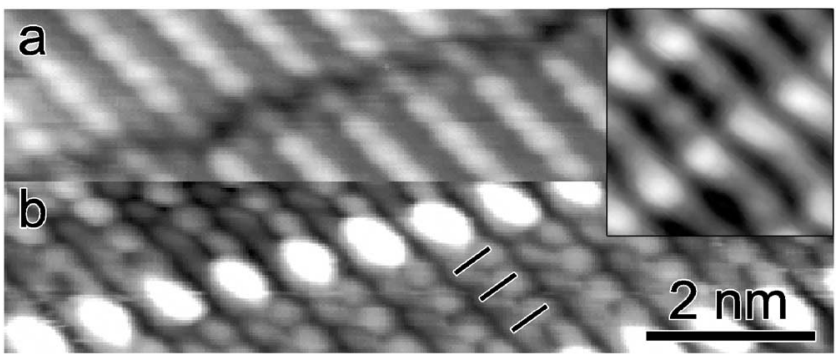

FIG. 4. (a) Empty-state and (b) filled-state dual-bias STM image of the $\mathrm{Si}(112) 6 \times 1-\mathrm{Ga}$ surface. In (b) the ladder structure is indicated. Tunneling conditions: $\pm 1 \mathrm{~V}, 0.05 \mathrm{nA}$. The inset shows a filled-state image with a slightly lower resolution. Tunneling conditions: $-2 \mathrm{~V}, 0.1 \mathrm{nA}$. 
the $\mathrm{Si}(112) 6 \times 1-\mathrm{Ga}$ surface is at variance with the new experimental observations. Consequently, the intuitive idea of metal adatom step-edge decoration does not seem applicable for the $\mathrm{Ga} / \mathrm{Si}(112)$ interface.

\section{STM-IMAGE SIMULATIONS}

Extensive DFT calculations were performed to identify the precise atomic structure of the $\operatorname{Si}(112) 6 \times 1-G a$ surface. Using the DFT local-orbital Hamiltonian of the surface together with nonequilibrium Keldysh-Green-function techniques, ${ }^{28,29}$ we calculated theoretical STM images of these most promising structures, which were then compared with the high-resolution experimental STM images. The different $\operatorname{Si}(112) 6 \times 1-G a$ structures analyzed in this paper, with Ga coverages ranging from 5 to $11 \mathrm{Ga}$ atoms per $6 \times 1$ unit cell, have been generated starting from the JKP model (see Fig. 1) in the following way: (i) replacing some of the $\mathrm{Si}$ atoms in the Si-dangling-bond row by $\mathrm{Ga}$ atoms (hereafter referred to as $\mathrm{Ga}$ terrace atoms); (ii) replacing some of the $\mathrm{Ga}$ atoms at the step edge by $\mathrm{Si}$ atoms; (iii) considering also the replacement of $\mathrm{Si}$ or $\mathrm{Ga}$ atoms on the step-edge and terrace rows by vacancies and the addition of $\mathrm{Ga}$ or $\mathrm{Si}$ atoms in the vacancy lines. In total, more than 40 new structures were fully relaxed, their surface energies and electronic structures calculated, and their corresponding theoretical STM images obtained. In the following, we compare the theoretical STM images of structures with the lowest total energies; a detailed chemical potential analysis of the total energies is deferred to Sec. VI.

Figures 5(a) and 5(b) show two examples of simulated STM images for some of these structural models, with a top view of the corresponding atomic structure superimposed. Figure 5(a) corresponds to a structural model that contains, per $6 \times 1$ unit cell, six Ga atoms in the terrace row and five $\mathrm{Ga}$ atoms plus a vacancy in the step-edge row; in Fig. 5(b) there are five $\mathrm{Ga}$ and one $\mathrm{Si}$ in the terrace row and five $\mathrm{Ga}$ plus a vacancy in the step-edge row. The simulated STM images for the different structural models are compared in detail with the experimental high-resolution STM images. For example, Fig. 5(a) (filled state) is similar to the filledstate STM image obtained in Ref. 20; also the empty-state image of Fig. 5(b) is in good agreement with the experimental STM image shown in Fig. 3. However, a detailed analysis of both empty-state and filled-state images, as well as registry-aligned dual images (e.g., Fig. 4) reveal that these models present some inconsistency with the experimental high-resolution information. For example, the structural model of Fig. 5(a) is symmetric with respect to the vacancy line, which is in disagreement with Fig. 3; in the case of Fig. 5(b) registry-aligned dual-bias STM images show that the bright protrusion in the filled-state image is located in the vacancy line, aligned with the brighter Ga row of the emptystate image, while in the simulated filled-state image, it appears in between the two Ga rows.

Thus, a detailed comparison of the theoretical STM images for the different structural models with the experimental STM images was performed. From this analysis, we concluded that the correct atomic model for the $\operatorname{Si}(112) 6 \times 1$ -

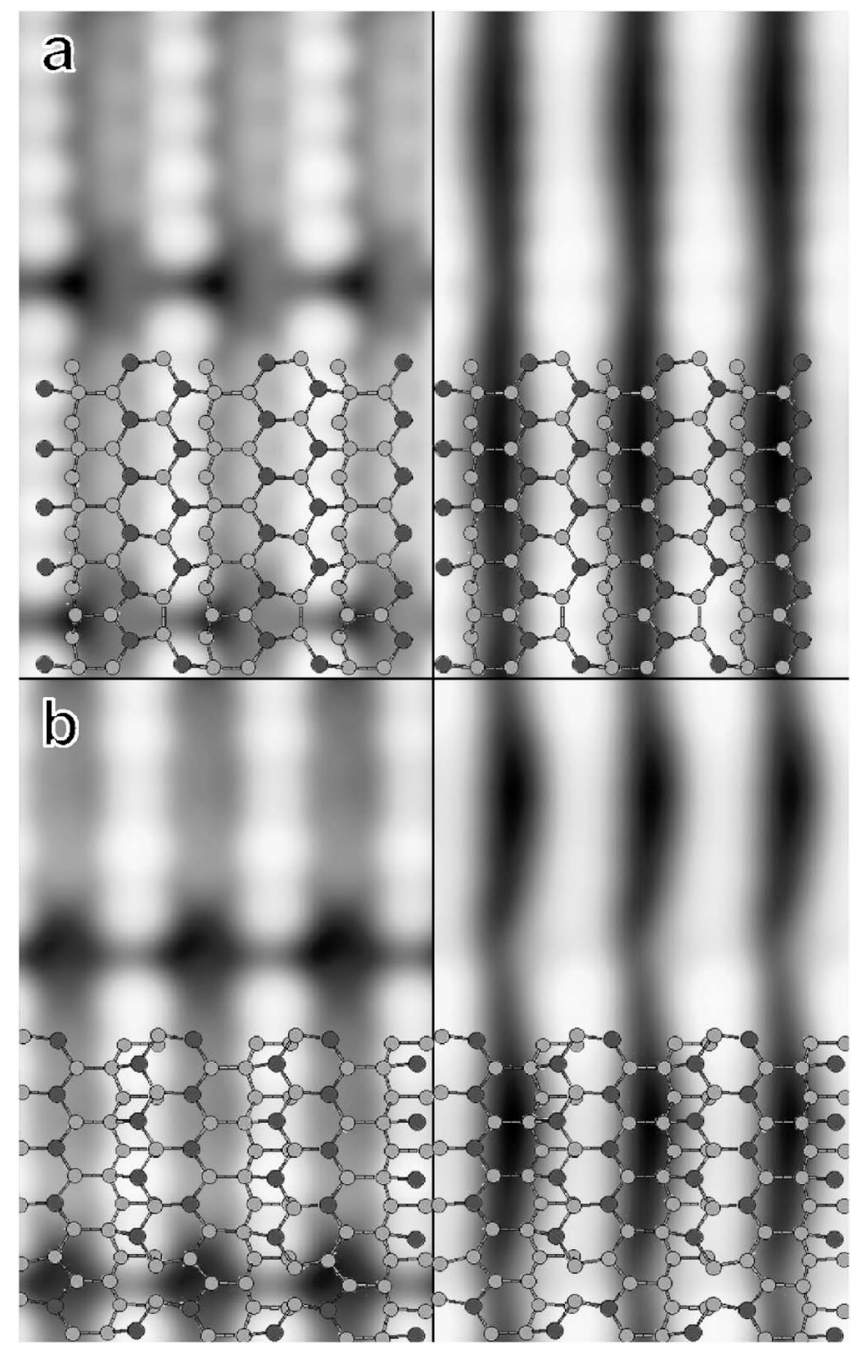

FIG. 5. Simulated empty-state (left) and filled-state (right) STM images of some of the structural models analyzed, with a ball-andstick representation (top view) superimposed on top of the STM images. (a) structural model with, per $6 \times 1$ unit-cell, six Ga atoms in the terrace row and five Ga atoms in the step-edge row; (b) a structural model with, per $6 \times 1$ unit-cell, five $\mathrm{Ga}$ and one $\mathrm{Si}$ in the terrace row, and five $\mathrm{Ga}$ in the step-edge row. $\mathrm{Ga}$ atoms: dark; $\mathrm{Si}$ atoms: light.

Ga is the one shown in Fig. 6. In this new structural model, there are $10 \mathrm{Ga}$ atoms per $6 \times 1$ unit cell (to be compared with the RBS determination of $9 \pm 1 \mathrm{Ga}$ atoms), forming two parallel rows, in a zigzag configuration (see also Fig. 3). The upper row of step-edge $\mathrm{Ga}$ atoms adsorbed at the (111)-like step is equivalent to the Ga row in the JKP model. But the $\mathrm{Si}$ dangling bond row in the JKP model has been replaced by a second row of $\mathrm{Ga}$ atoms (henceforth, the "terrace Ga row"). Each $\mathrm{Ga}$ row contains five $\mathrm{Ga}$ atoms per $6 \times 1$ unit cell, i.e., there is a Ga vacancy in each row. These vacancies are placed at adjacent sites in the (zigzag) two rows, giving rise to an asymmetry in the vacancy line (see Figs. 6 and 3).

Inside the vacancy lines, missing $\mathrm{Ga}$ atoms expose the underlying $\mathrm{Si}$ atoms. These $\mathrm{Si}$ atoms rebond forming $\mathrm{Si}-\mathrm{Si}$ dimers on the terraces and $\mathrm{Si}-\mathrm{Ga}$ dimers along the step edges in each unit cell. Specifically, by rotating a step-edge $\mathrm{Si}$ atom 


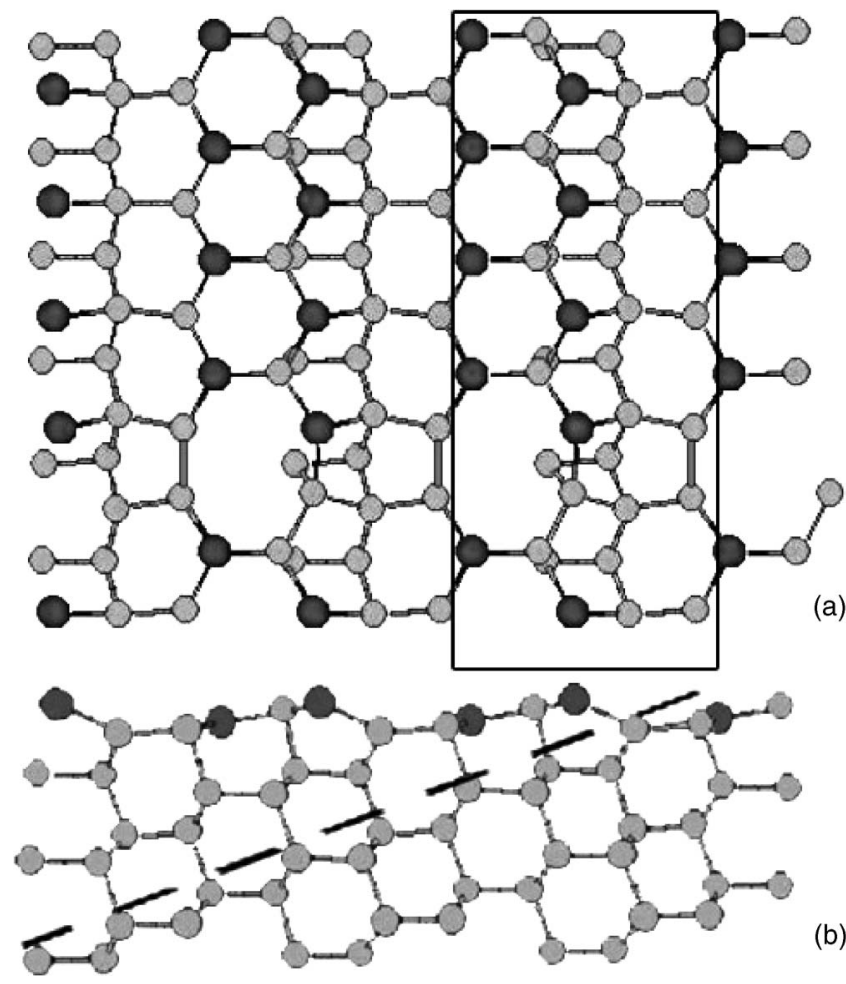

FIG. 6. Ball-and-stick representation of the energy minimized structure for the $\mathrm{Si}(112) 6 \times 1-\mathrm{Ga}$ surface; top view (a) and side view (b). In (a) a $6 \times 1$ unit cell is indicated and in (b) a (111) plane is indicated. Si atoms: light; $\mathrm{Ga}$ atoms: dark.

toward the step-edge Ga row, this $\mathrm{Si}$ atom can rebond to two neighboring $\mathrm{Si}$ atoms and a step-edge $\mathrm{Ga}$ atom, forming a $\mathrm{Si}-\mathrm{Ga}$ dimer with the latter. The rebonding of the $\mathrm{Si}$ atoms in the vacancy line also implies that the Ga vacancies on both $\mathrm{Ga}$ rows must be aligned. In contrast with the tetravalent $\mathrm{Si}$ atoms in the step-edge decorated JKP model, both the trivalent $\mathrm{Ga}$ atoms in the threefold adsorption sites on the (111)like terraces, and the exposed $\mathrm{Si}$ atoms inside the vacancy lines present no unsaturated dangling bonds; the resulting structure (Fig. 6) is fully passivated and the surface is semiconducting.

The calculated theoretical STM images corresponding to this zigzag model are shown in Fig. 7, with a top view of the structural model superimposed on top. Both the empty-state and filled-state images are in excellent agreement with the experimental ones (see Fig. 3). It shows that the two atom rows imaged in the empty-state STM images are indeed the step-edge $\mathrm{Ga}$ row and the terrace $\mathrm{Ga}$ row, ruling out the formation of a Ga-atom step-edge decorated structure. In addition, the asymmetry in the vacancy lines observed experimentally in the empty state is neatly reproduced in the simulated STM images. In the simulated filled-state image, fuzzy lines with a big, symmetric protrusion inside the vacancy line are observed, which are in agreement with the experimental images. Figure 7(b) clearly shows that the big protrusion corresponds to the Ga-Si dimer. Furthermore, it shows that the fuzzy lines are originating from a $\mathrm{Si}-\mathrm{Ga}$ bond on the (111)-like terrace. They form a ladder configuration, in agreement with the atomic-resolution experimental image in

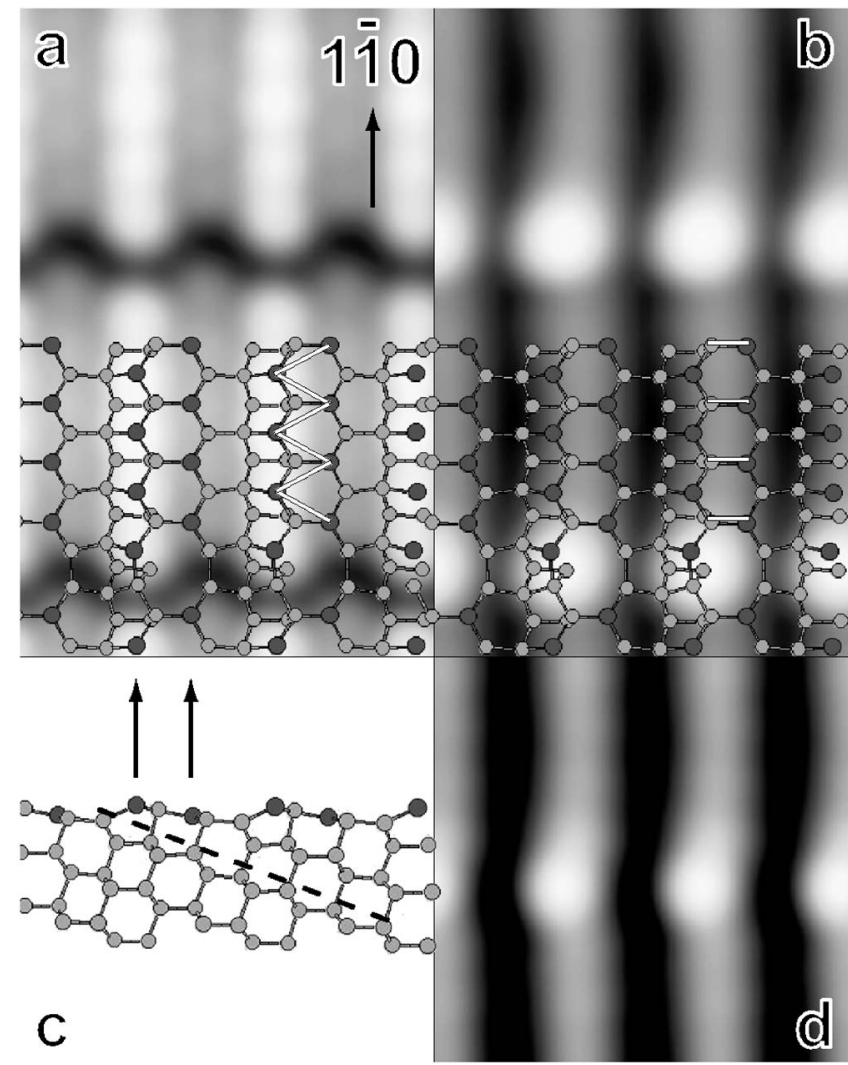

FIG. 7. Simulated empty-state (a) and filled-state (b) STM images of the zigzag model of Fig. 6. A top view of the ball-and-stick representation is superimposed on top of the STM images. Ga atoms: dark; Si atoms: light. Zigzag symmetry and ladder symmetry indicated with white bars in (a) and (b), respectively. Tunneling bias $2 \mathrm{~V}$ (a) and $-1.3 \mathrm{~V}$ (b). (c) Side view of the proposed model. The (111) plane is indicated with a dotted line. (d) Simulated filled-state image, $-2 \mathrm{~V}$.

Fig. 4. The only feature which was not reproduced is the slightly higher apparent height, in the empty-state experimental images, of the Ga atoms in the two terrace Ga rows directly adjacent to the vacancy line. Finally, we mention that changing the tunneling conditions in the simulated STM images (tip-sample distance, voltage), the experimental STM images of Ref. 20 can be recovered, as shown in Fig. 7(d).

\section{SPECTROSCOPY}

We also have studied this surface reconstruction with scanning tunneling spectroscopy. While imaging the surface with a constant tunneling current, at every third data point an $I-V$ curve is measured with the feedback loop switched off during this $I-V$ measurement. In Fig. 8, we have averaged $I$ - $V$ curves measured on the upper atom rows, the lower atom rows, and on the vacancy lines separately (the respective areas being determined from the empty-state STM image). This results in three curves, representing the electronic structure on the terrace and step-edge Ga rows and the electronic structure inside the vacancy line. At bias voltages below the bulk conduction band minimum, the tunneling current is limited by thermionic emission, as is evident from the linear 


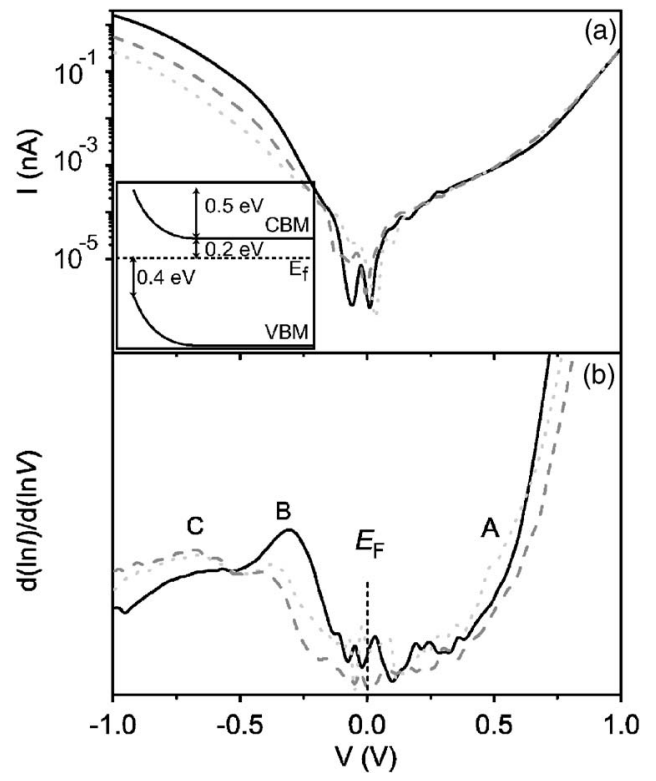

FIG. 8. Semilogarithmic (a) and normalized derivative (b) plots of $I-V$ curves averaged over the step-edge (dotted) and terrace (dashed) atom rows, and the vacancy lines (solid), respectively. STS set point: $1 \mathrm{~V}, 0.3 \mathrm{nA}$. The inset in (a) shows the band structure inferred from the data.

increase of the $\log _{10}(I)-V$ curve, ${ }^{33}$ up to the conduction-band minimum (CBM) at $0.7 \mathrm{~V}$ [see Fig. 8(a)]. Consequently, the bulk valence-band maximum (VBM) at the surface should be located at $\sim-0.4 \mathrm{~V}$, implying an upward band bending of $\sim 0.5 \mathrm{eV}$ for this $n$-type specimen $\left(10^{15} \mathrm{~cm}^{-3}\right), E_{f}-E_{V B M}$ and $E_{C B M}-E_{f}$ being $\sim 0.4$ and $\sim 0.7 \mathrm{eV}$ at the surface, respectively, as shown in the inset of Fig. 8(a). These data are consistent with the measured surface photovoltage in Refs. 22 and 34. In Fig. 8(b), we have plotted the normalized derivative of the three $I-V$ curves (i.e., $\partial \ln I / \partial \ln V$ ), originating from the three different areas within the unit cell. These tunneling spectra are proportional to the local density of states (LDOS) at the respective areas of the sample surface over which the averaging took place (see Refs. 35-37). In all curves, there is no density of states (DOS) at the Fermi level, but a gap of exists between the filled-state and empty-state bands showing that indeed the surface is semiconducting. The tunneling spectra on the two Ga atom rows appears to be similar in shape, whereas the tunneling spectra inside the vacancy line deviates from the former two. The leading edge of the total tunneling spectrum in the filled-state spectrum arises from a state $(B)$ that is located mainly inside the vacancy lines at $\sim-0.3 \mathrm{eV}$. The two Ga rows feature a broadfilled state at higher binding energy $(C)$. In the empty-state tunneling spectra, the two atom rows show a small shoulder (A) at $\sim 0.6 \mathrm{eV}$, just below the bulk CBM. Thus, a surface band gap of $\sim 0.9 \mathrm{eV}$ is deduced. These experimental data are entirely consistent with the presence of two equivalent, threefold coordinated rows of Ga atoms at these positions, fully passivating the surface. Notice that we inferred the surface band gap from the separation between peaks $A$ and $B$ using the peak position or centroids and not the onsets. The justification for this procedure comes from a detailed com-

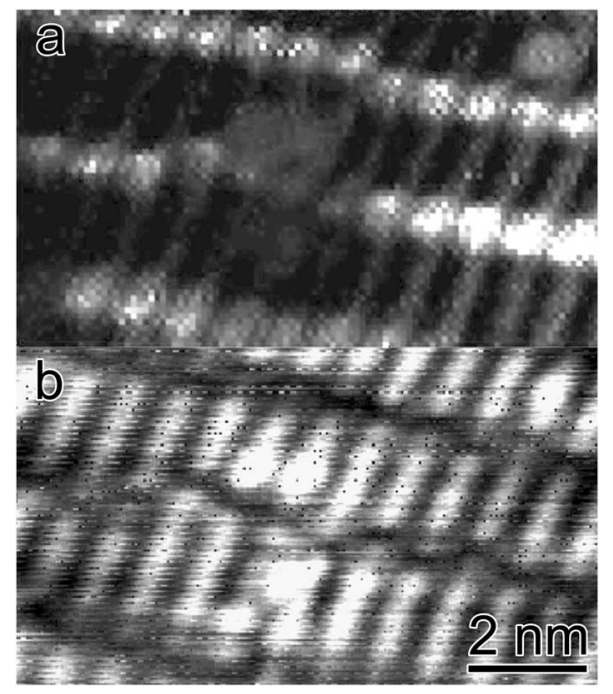

FIG. 9. (a) $\partial I / \partial V$ map of measured STS curves at $-0.31 \mathrm{~V}$. STS set point: $1 \mathrm{~V}, 0.3 \mathrm{nA}$. (b) Corresponding topographic STM image. Tunneling conditions: $1 \mathrm{~V}, 0.3 \mathrm{nA}$.

parison with theoretical STS data, as will be discussed below.

In addition, we have constructed a $\partial I / \partial V$ map of the STS measurements. In a $\partial I / \partial V$ map, the value of the derivative of the $I-V$ curves at a certain voltage $V$ is plotted as a twodimensional image, with the $x$ and $y$ coordinates corresponding to the topographic STM image. In Fig. 9(a), the derivative of the $I-V$ curves at $-0.31 \mathrm{~V}$ is plotted; and the corresponding empty-state STM image is shown in Fig. 9(b). Indeed, the largest slope in the $I-V$ curves at $-0.31 \mathrm{~V}$ is located inside the vacancy lines (i.e., here the largest increase in tunneling current is observed, corresponding to the largest LDOS as compared to the LDOS at this specific energy at other locations on the surface). Only a very small intensity variation is observed perpendicular to the atom rows, consistent with the similar filled-state tunneling spectra on the two Ga rows in Fig. 8(a).

From the structural model and its spatially resolved DOS, theoretical STS curves were calculated [see Fig. 10(b)]. As for the STM images, these results are obtained using the LO-DFT FIREBALL96 Hamiltonian of the surface and the Keldysh-Green-function approach. The corresponding calculated LDOS of the zigzag model, averaged over different areas is shown in Fig. 10(a) (a broadening of $0.1 \mathrm{eV}$ has been used). The calculated STS curves shown in Fig. 10(b) were obtained by placing the tip over the respective areas, calculating the current as a function of a voltage sweep using the calculated LDOS (Fig. 10), and averaging over the areas of interest. The FIREBALL96 local-orbital calculations employ a minimal basis set, resulting typically in band gaps that are too large. Nonetheless, excellent qualitative agreement exists between the calculated STS curves and normalized derivatives of the experimental STS curves [Fig. 8(b)]. The calculated LDOS and STS curves confirm that the large peak $B$ just beneath the band gap indeed is mainly associated with states that are located on the $\mathrm{Si}-\mathrm{Ga}$ dimer inside the vacancy lines. The two Ga rows contribute almost equally to a broad peak in the DOS at higher binding energy $(C)$ and a small 


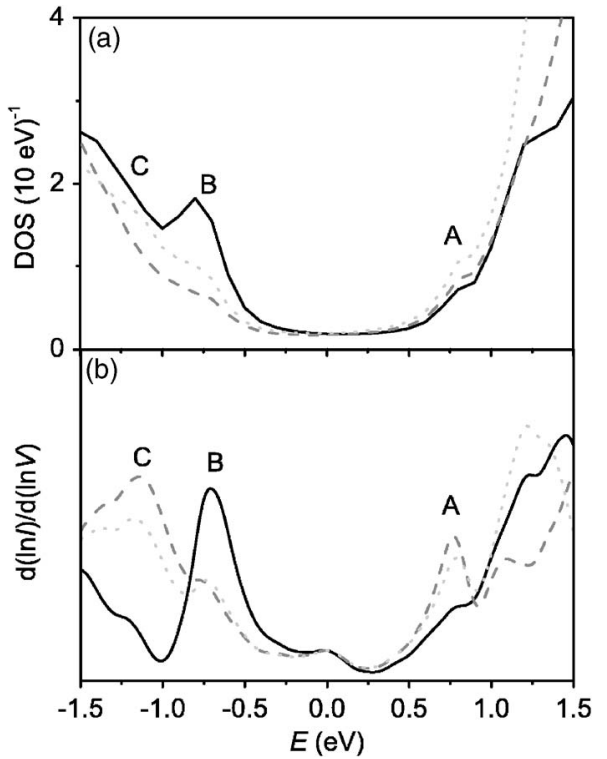

FIG. 10. (a) Calculated LDOS (FIREBALL96) averaged over the step-edge (dotted) row, terrace (dashed) Ga row, and vacancy line (solid), respectively. The LDOS at the vacancy line is calculated as the average density of states of the $\mathrm{Ga}-\mathrm{Si}$ dimer and $\mathrm{Si}-\mathrm{Si}$ dimer. (b) Normalized derivative of the $I-V$ curves shown in (a). A broadening of $0.1 \mathrm{eV}$ has been applied. Note the larger energy scale as compared to Fig. 8 due to the overestimation of the gap in the local-orbital calculation.

shoulder in the DOS just above the band gap $(A)$, in full agreement with the normalized derivative of the tunneling spectra in Fig. 8(b). The empty state DOS is very similar for both Ga rows. Consequently, the $\sim 0.4 \AA$ higher appearance of the $\mathrm{Ga}$ atoms at the step edge in the empty-state image is due to their on-average higher-atomic positions, and, thus, the empty-state STM image reflects the real surface topography at these voltages.

We have calculated the surface band structure using both the LO and PW-DFT methods, within the local-density approximation (LDA) for exchange-correlation contributions. While the LO calculation overestimates the value of the bulk band gap, the PW calculation typically underestimates the band gap. In the LO band structure (not shown) a surfacestate band gap of $1.2 \mathrm{eV}$ is obtained between the states $A$ and $B$, while the separation between peaks $A$ and $B$ in the calculated STS is close to $1.4 \mathrm{eV}$. This suggests that in order to measure the band gap, it is reasonably accurate to use the peak positions instead of the (poorly defined) peak onsets in the experimental $\partial \ln I / \partial \ln V$ curves. The observed splitting between $A$ and $B$ in the experimental curves is $\sim 0.9 \mathrm{eV}$ indicating that the experimental band gap is $\sim 0.8 \mathrm{eV}$. Figure 11 shows the band structure as calculated with the PW-DFT code, showing a surface band gap of $0.77 \mathrm{eV}$ between states $A$ and $B .{ }^{40}$ The calculations place filled state $B$ slightly above the VBM, which is in agreement with the experimental observation. On the other hand, the empty surface state $A$ is located at or slightly above the CBM according to the PWDFT calculations; while, experimentally, state $A$ appears slightly below the bulk CBM [see Fig. 8(b)]. The PW gap of $0.77 \mathrm{eV}$ is comparable to the experimental band gap of

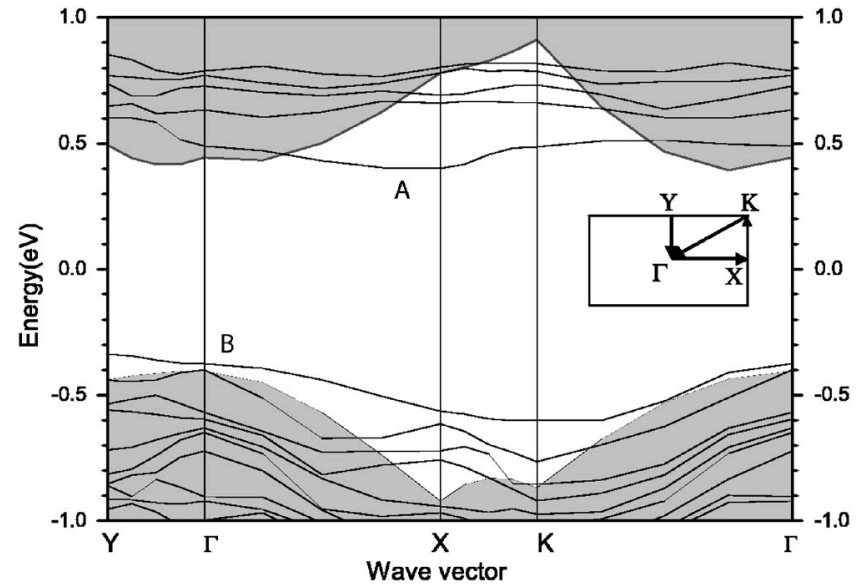

FIG. 11. Calculated band structure (CASTEP) of the zigzag structural model. Surface states labeled $A$ and $B$ are mentioned in the text. The shaded area shows the bulk projected band structure of the Si substrate. The inset shows the surface Brillouin zone probed.

$0.8 \mathrm{eV}$. However, the precise location of state $A$ in the calculations directly affects the value of the band gap.

Despite the fact that the $\mathrm{Ga} / \mathrm{Si}(112)$ overlayer appears to be quasi-two-dimensional(2D) in atomic structure, the electronic structure of this overlayer is quasi-one-dimensional. The Ga-atom-induced surface band $A$ disperses around the $X$-point minimum in the upper part of the band gap. The dispersion of this band near the $X$ point yields an effective mass of $m^{*} \sim 1.48 m_{e}$ along the $X-\Gamma$ direction, and $m^{*}$ $\sim 0.15 m_{e}$ along $X-K$. This indicates a quasi-one-dimensional dispersion. This Ga band is initially empty, but could perhaps be populated in a controllable way using a biased-gate electrode or a heavily $n$-type doped substrate, making the $\mathrm{Si}(112) 6 \times 1-\mathrm{Ga}$ surface a promising system for the experimental study of electron transport in one-dimensional atomic wires.

\section{COMPETING STRUCTURES}

The discussion above shows that, to elucidate the precise atomic structure of a complex surface like $\mathrm{Si}(112) 6 \times 1-\mathrm{Ga}$ from the comparison of theoretical and experimental STM images, it is necessary to use high-resolution experimental STM images, including registry-aligned dual-bias information and STS data, combined with state-of-the-art theoretical STM simulations. These simulations were performed on the subset of possible structures that were deemed most realistic on the basis of total energy considerations. In this section, we explore the relative stability of the various structures, which gives a more physical basis to the proposed structural model.

In general, the precise stoichiometry of the surface is not known, and, thus, the analysis of the relative stability of different structural models requires the calculation of the surface energy $F$ as a function of the different chemical potentials.

\section{A. Chemical potential analysis}

For the analysis of the relative stabilities of the various structures, we need to calculate the surface energy $F=E_{t o t}$ 


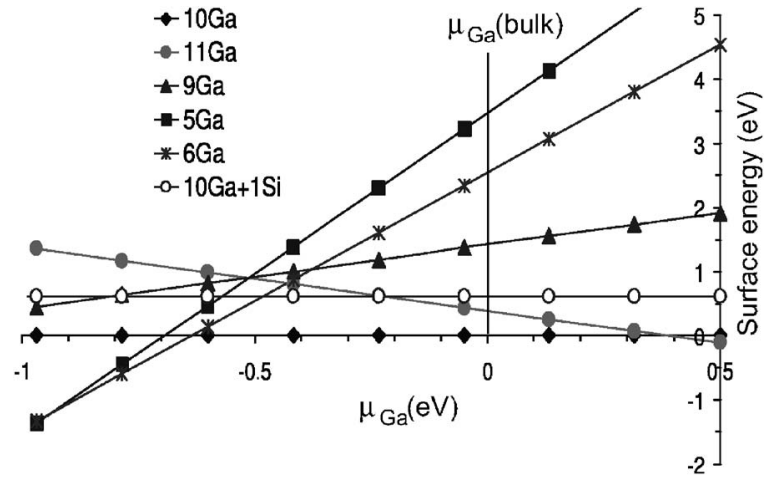

FIG. 12. Surface energy as a function of the Ga chemical potential (CASTEP). The Ga chemical potential is plotted relative to the chemical potential in bulk Ga. 5Ga (filled squares) is the step-edge decorated JKP model.

$-\mu_{G a} N_{G a}-\mu_{S i} N_{S i}$, where $E_{\text {tot }}$ is the total energy per unit cell; $\mu_{G a}, \mu_{S i}$ are the Ga and Si chemical potentials; and $N_{G a}, N_{S i}$ are the number of $\mathrm{Ga}$ and $\mathrm{Si}$ atoms in the unit cell. For $\mu_{S i}$, we use the total energy (per atom) of bulk Si (i.e., the surface is in equilibrium with the substrate). The value of $\mu_{G a}$ is not determined by the substrate, but it can be estimated analyzing the experimental conditions (see below).

Figure 12 shows the surface energy $F=E_{t o t}-\mu_{G a} N_{G a}$ $-\mu_{S i} N_{S i}$ as a function of $\mu_{G a}$. In this figure, we use the structural model of Fig. 6 as a reference and plot $F$ for some of the most promising models, as calculated with the PW code (CASTEP). In order to estimate the value of $\mu_{G a}$ we have to analyze the experimental conditions of the Ga deposition. In the one-step process the $(6 \times 1)$-phase is formed under a $\mathrm{Ga}$ flux from the effusion cell with the sample held at a temperature of $T=825 \mathrm{~K}$. At this temperature, the incoming flux of $\mathrm{Ga}$ atoms is balanced by a flux of Ga atoms desorbing from the surface, thus establishing a quasiequilibrium. This allows us to relate the chemical potential $\mu_{G a}$ in the effusion cell with the chemical potential $\mu_{G a}$ at the sample. The chemical potential in the effusion cell may be approximated by the total energy of bulk Ga, $\mu_{G a}$ (bulk), i.e., the Ga vapor in the effusion cell is in equilibrium with the solid. ${ }^{41}$

Considering also the equilibrium between the sample and the Ga vapor in contact with the sample, we can estimate the chemical potential at the sample

$$
\mu_{G a}=\mu_{G a}(\text { bulk })-k_{B} T \ln \left(\frac{p_{c}}{p_{s}}\right),
$$

where $p_{c}$ is the Ga vapor pressure in the effusion cell and $p_{s}$ the Ga vapor pressure at the sample. Since the effusion cell flux is proportional to its vapor pressure $p_{c}$ times the cell aperture area, and the sample flux is also proportional to its corresponding vapor pressure $p_{s}$ times the sample area, we conclude that $\left(p_{c} / p_{s}\right) \sim 10^{2}\left(10^{3}\right)$, and $\mu_{G a}=\mu_{G a-\text { bulk }}$ $-0.32(0.48) \mathrm{eV}$. In Fig. 12, we see that for this range of $\mu_{G a}$ the structural model of Fig. 6 presents the lowest surface energy $F$. This result strongly supports our conclusion that the structural model for the $\operatorname{Si}(112) 6 \times 1-G a$ surface is the one depicted in Fig. 6.
Comparing our model with the step-edge decorated JKP model, an important difference is that the new structural model (Fig. 6) presents no partially filled dangling bonds as discussed above. The stability of the new model is related to the full passivation of the substrate, removing all dangling bonds, and the associated decrease in surface free energy. The results shown in Fig. 12 suggest, however, that the JKP model might be stabilized for very low $\mu_{G a}$ values. We should stress that our theoretical analysis has been directed to search for surface atomic structures that could explain the experimental results (STM and RBS) for the $\mathrm{Si}(112) 6 \times 1$ Ga surface; thus, surface structures with lower Ga coverages that should be favored for low $\mu_{G a}$ values have not been analyzed as thoroughly as those with coverages close to 9-10 $\mathrm{Ga}$ atoms $/ 6 \times 1$ unit cell. Nevertheless, we may perform a simple analysis, comparing the surface energy of the stepedge decorated JKP model, with the surface energy of a simple hypothetical surface: half the surface is covered with the structure of Fig. 6 (i.e., both step-edge and terrace Ga rows), while the other half consists of clean $\mathrm{Si}(112)$. Both the step-edge decorated JKP system and this hypothetical halfhalf case present the same Ga coverage and, thus, the same behavior of $F$ as a function of $\mu_{G a}$ (i.e., the same slope in Fig. 12). This comparison reveals that the hypothetical case is lower in energy [by $\sim 0.7 \mathrm{eV} /(10 \mathrm{Ga}$ atoms $)]$, for all $\mu_{G a}$ values, showing that the step-edge decorated case is unlikely to be stabilized at lower Ga coverage, and phase separation into bare $\mathrm{Si}(112)$ and the $\mathrm{Si}(112) 6 \times 1-\mathrm{Ga}$ zigzag surface will occur instead. Note that the faceting of the unstable Si(112) surface, which was not accounted for in this calculation, would increase this energy difference, making phase separation even more favorable compared to the step-edge decorated JKP model.

Another possible scenario for obtaining a step-edge decorated $\mathrm{Ga}$ row would be to use the experimentally observed fully passivated $\mathrm{Si}(112) 6 \times 1-$ Ga surface (Fig. 6) as starting point and try to kinetically stabilize a metastable step-edge decorated structure by selectively desorbing the $\mathrm{Ga}$ atoms from the terraces. Experimentally, this might happen in the two-step preparation procedure (see Sec. II). We have studied this possibility by calculating desorption energies of terrace and step-edge $\mathrm{Ga}$ atoms from the $\mathrm{Si}(112) 6 \times 1-\mathrm{Ga}$ surface (Fig. 6). In particular, we have considered removing the $\mathrm{Ga}$ atoms close to the vacancy line as well as the replacement of those $\mathrm{Ga}$ atoms by $\mathrm{Si}$ atoms. In both cases, the desorption energies are lower by $\sim 0.7 \mathrm{eV}$ for step-edge $\mathrm{Ga}$ atoms than for $\mathrm{Ga}$ atoms on the terraces. This result suggests that a metastable step-edge decorated structure likely cannot be achieved by thermally desorbing the Ga terrace atoms.

\section{B. Intrinsic structural disorder}

In the experimental STM images, the vacancy lines are not exactly straight, but some meandering is observed, as shown in Fig. 13 [see also Figs. 2(b) and 3]. The new structural model is able to fully explain the experimentally observed meandering. ${ }^{12}$ It was proposed by Erwin et al. ${ }^{21}$ that this meandering of the vacancy lines could be explained by the coexistence of $6 \times 1$ and $5 \times 1$ unit cells on the surface. 


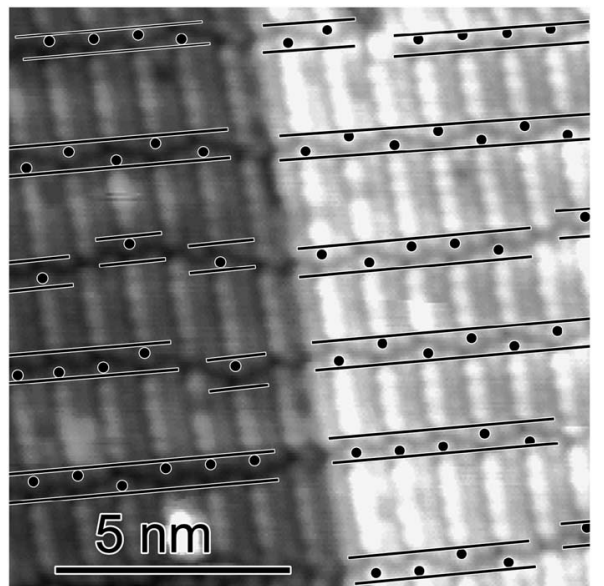

FIG. 13. Detailed STM image, showing the two contributions to the meandering of the vacancy lines. Straight lines are drawn through domains with unit cells of the same size. Occasional jumps in the lines are due to different unit cell sizes, as explained in the text. Dots are placed on the $\mathrm{Ga}$ atom of the $\mathrm{Ga}-\mathrm{Si}$ dimer at the vacancy of the step-edge rows, showing the fluctuations due to the intrinsic disorder of the random orientation of the $\mathrm{Ga}-\mathrm{Si}$ dimer.

This results in occasional steps in the vacancy lines, equivalent to the observed meandering of the dimer-vacancy lines on the Ge-covered $\operatorname{Si}(001)$ surfaces. ${ }^{38}$ We have analyzed the stability of the zigzag structural model as a function of the longitudinal periodicity. Figure 14 shows the surface energies $F$ of this model for different periodicities: $5 \times 1,6 \times 1$, and $7 \times 1$. The $5 \times 1$ surface corresponds to four $\mathrm{Ga}$ atoms in each $\mathrm{Ga}$ row between vacancy lines, while the $7 \times 1$ surface presents six Ga atoms in each row between vacancy lines. For our estimated range of $\mu_{G a}$, the $6 \times 1$ surface presents the lowest $F$, while the $5 \times 1$ is only $0.1-0.2 \mathrm{eV}$ higher per 6 $\times 1$ unit cell. This small energy difference should lead to the experimental observation of $5 \times 1$ unit cells. Indeed these unit cells are frequently observed in the experimental images (see Fig. 13). Thus, it appears that the predictions from the 1D Frenkel-Kontorova model regarding the vacancy-line

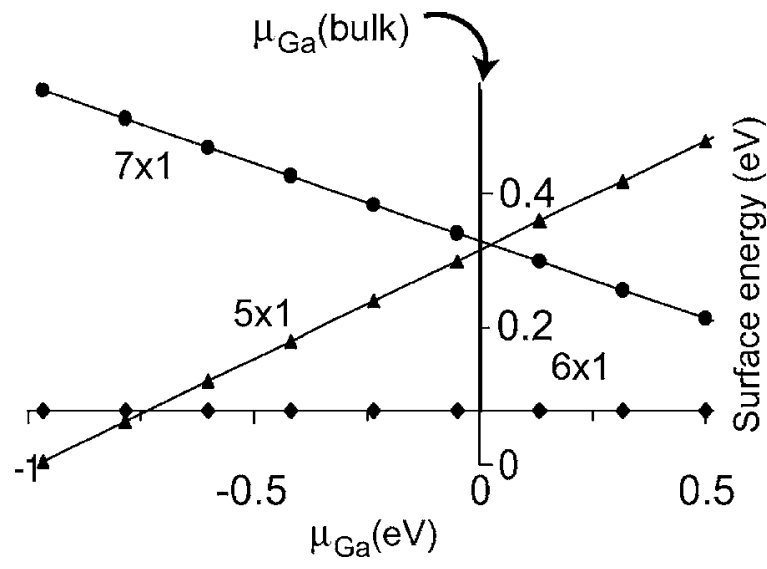

FIG. 14. Free energy as a function of the Ga chemical potential for $5 \times 1,6 \times 1$ and $7 \times 1$. The Ga chemical potential is plotted relative to the chemical potential in bulk Ga. Note the difference in scale on the abscissa as compared to Fig. 12. spacing in the step-edge decorated structure ${ }^{21}$ also apply to the quasi-2D zigzag arrangement of $\mathrm{Ga}$ atoms presented here. This conclusion is not very surprising as it was concluded in Ref. 21 that the strain induced by the size difference of $\mathrm{Ga}$ and $\mathrm{Si}$ completely dominates the energetics of the periodicities. Apparently this conclusion still holds when an extra Ga row is added.

However, careful investigation of the experimental images reveals that the meandering of the vacancy lines as observed in Fig. 2 is not only due to the competing longitudinal periodicities. Instead, for large sections of the surface, the terrace $G a$ rows are perfectly periodic in the $\times 1$ direction with $(n-1) \mathrm{Ga}$ atoms per terrace $\mathrm{Ga}$ row in the $n \times 1$ unit cell. But in these ordered sections, the number of $\mathrm{Ga}$ atoms in the step-edge Ga rows appears to fluctuate between $n-2$ and $n$. The proposed structural model perfectly explains these intrinsic fluctuations (i.e., fluctuations within a $n \times 1$ domain); they are related to the orientation of the $\mathrm{Si}-\mathrm{Ga}$ dimer in the step-edge rows. The twofold symmetry of the 112 substrate in the $[1 \overline{1} 0]$ direction is broken by the $\mathrm{Si}-\mathrm{Ga}$ dimers, resulting in two degenerate orientations of these dimers. The energy associated with interchanging the atoms of a $\mathrm{Si}-\mathrm{Ga}$ dimer has been calculated ${ }^{12}$ to be less than $10 \mathrm{meV}$ per $12 \times 1$ unit cell. This small energy difference explains the appearance of frequent meandering in the aligned vacancies in the step-edge Ga rows, thus accounting for the majority of the fluctuations in the vacancy lines observed in the experimental images. The absence of these fluctuations in the filled-state images [compare Figs. 4(a) and 4(b) and also Figs. 9(a) and 9(b)] is the result of the fact that the bright protrusion in the vacancy line appears in the center of the $\mathrm{Ga}$-Si dimer, making its appearance insensitive to the orientation of the $\mathrm{Ga}-\mathrm{Si}$ dimer.

\section{SUMMARY AND CONCLUSIONS}

The $6 \times 1$ reconstruction of $\mathrm{Ga}$ on vicinal $\mathrm{Si}(112)$ was studied with STM, STS, RBS, and extensive DFT calculations. High-resolution STM experiments revealed an asymmetry in the vacancy lines of the $\mathrm{Si}(112) 6 \times 1-\mathrm{Ga}$ surface that is inconsistent with the JKP model of step-edge decoration. STS measurements also rule out formation of quasi-1D metal wires while RBS experiments indicated a Ga coverage twice as large as was previously inferred from the JKP model. Extensive DFT calculations were used to analyze the relative stability of more than 40 structures, taking the chemical potential of the $\mathrm{Ga}$ adsorbate into account. Theoretical STM images were calculated for the most promising structures and compared in detail with the experimental STM images.

A new structure emerged containing ten $\mathrm{Ga}$ atoms per 6 $\times 1$ unit cell. The Ga atoms decorate the step edge and passivate the terrace atoms, thereby forming a zigzag pattern. Excellent agreement between experimental and theoretical STM and STS data confirmed the validity of the proposed zigzag model and demonstrate the power of such a comparison. Ga atoms are threefold coordinated and $\mathrm{Si}$ dangling bonds are all passivated so the surface is semiconducting. The "broken-bond orbitals" inside the vacancy lines rebond 
to form $\mathrm{Si}-\mathrm{Ga}$ and $\mathrm{Si}-\mathrm{Si}$ dimers. The observed meandering of the vacancy lines originates from thermal fluctuations between the two symmetry-degenerate orientations of the $\mathrm{Si}$ Ga dimer, in conjunction with thermal fluctuations between competing $6 \times 1$ and $5 \times 1$ units.

While step-edge decoration of vicinal metal surfaces works, ${ }^{39}$ the observed drive toward chemical passivation suggests that step-edge decoration of vicinal semiconductors is not a viable method to produce 1D metal wires. Although this general conclusion remains to be tested further, it is clear that partially filled dangling bonds on the terraces of vicinal surfaces are always greatly reduced in number or eliminated altogether in the reconstruction.

As shown in this paper, predictive calculations along these lines should always take into account the chemical potential of the adsorbate. The latter depends on the experimental preparation conditions [e.g., see Eq. (4)]. Successful prediction of systems with perfect 1D metal adatom step-edge decoration could facilitate the quest for the experimental re- alization of Luttinger liquids in such systems, possibly enabling a convincing proof of spin-charge separation with angle-resolved photoemission spectroscopy.

\section{ACKNOWLEDGMENTS}

This work is part of the research program of the Stichting voor Fundamenteel Onderzoek der Materie (FOM), which is financially supported by the Nederlandse Organisatie voor Wetenschappelijk Onderzoek (NWO). This work was sponsored in part by the NSF under Grant No. DMR-0244570, the Ministerio de Ciencia y Tecnología (Spain) under Grant Nos. MAT2001-0665 and MAT2004-01271. One of us, S.R., wishes to acknowledge the Royal Netherlands Academy of Arts and Sciences. We thank T. M. Klapwijk for his stimulating support, and the AMOLF Institute in Amsterdam for performing the RBS experiments. Oak Ridge National Laboratory is managed by UT-Battelle, LLC, for the U.S. Department of Energy under Grant No. DE-AC-05-00OR22725.
*Corresponding author. Electronic address: p.c.snijders @ tnw.tudelft.nl

${ }^{1}$ H. W. C. Postma, M. de Jonge, Z. Yao, and C. Dekker, Phys. Rev. B 62, R10653 (2000).

${ }^{2}$ M. Grioni, I. Vobornik, F. Zwick, and G. Margaritondo, J. Electron Spectrosc. Relat. Phenom. 100, 313 (1999).

${ }^{3}$ J. Voit, Rep. Prog. Phys. 58, 977 (1994).

${ }^{4}$ F. J. Himpsel, K. N. Altmann, R. Bennewitz, J. N. Crain, A. Kirakosian, J.-L. Lin, and J. L. McChesney, J. Phys.: Condens. Matter 13, 11097 (2001).

${ }^{5}$ J. N. Crain, J. L. McChesney, F. Zheng, M. C. Gallagher, P. C. Snijders, M. Bissen, C. Gundelach, S. C. Erwin, and F. J. Himpsel, Phys. Rev. B 69, 125401 (2004).

${ }^{6}$ P. Starowicz, O. Gallus, T. Pillo, and Y. Baer, Phys. Rev. Lett. 89, 256402 (2002).

${ }^{7}$ P. Segovia, D. Purdie, M. Hengsberger, and Y. Baer, Nature (London) 402, 504 (1999).

${ }^{8}$ H. W. Yeom, S. Takeda, E. Rotenberg, I. Matsuda, K. Horikoshi, J. Schaefer, C. M. Lee, S. D. Kevan, T. Ohta, T. Nagao et al., Phys. Rev. Lett. 82, 4898 (1999).

${ }^{9}$ J. R. Ahn, H. W. Yeom, E. S. Cho, and C. Y. Park, Phys. Rev. B 69, 233311 (2004).

${ }^{10}$ O. Bunk, G. Falkenberg, J. H. Zeysing, L. Lottermoser, R. L. Johnson, M. Nielsen, F. Berg-Rasmussen, J. Baker, and R. Feidenhansl, Phys. Rev. B 59, 12228 (1999).

${ }^{11}$ I. K. Robinson, P. A. Bennett, and F. J. Himpsel, Phys. Rev. Lett. 88, 096104 (2002).

${ }^{12}$ C. González, P. C. Snijders, J. Ortega, R. Pérez, F. Flores, S. Rogge, and H. H. Weitering, Phys. Rev. Lett. 93, 126106 (2004).

${ }^{13}$ J. N. Crain, A. Kirakosian, K. N. Altmann, C. Bromberger, S. C. Erwin, J. L. McChesney, J.-L. Lin, and F. J. Himpsel, Phys. Rev. Lett. 90, 176805 (2003).

${ }^{14}$ J. R. Ahn, H. W. Yeom, H. S. Yoon, and I.-W. Lyo, Phys. Rev. Lett. 91, 196403 (2003).

${ }^{15}$ I. Matsuda, M. Hengsberger, F. Baumberger, T. Greber, H. W.
Yeom, and J. Osterwalder, Phys. Rev. B 68, 195319 (2003).

${ }^{16}$ T. Kanagawa, R. Hobara, I. Matsuda, T. Tanikawa, A. Natori, and S. Hasegawa, Phys. Rev. Lett. 91, 036805 (2003).

${ }^{17}$ T. M. Jung, R. Kaplan, and S. M. Prokes, Surf. Sci. 289, L577 (1993).

${ }^{18}$ T. M. Jung, S. M. Prokes, and R. Kaplan, J. Vac. Sci. Technol. A 12, 1838 (1994).

${ }^{19}$ J. E. Yater, A. Shih, and Y. U. Idzerda, Phys. Rev. B 51, 7365 (1995).

${ }^{20}$ A. A. Baski, S. C. Erwin, and L. J. Whitman, Surf. Sci. 423, L265 (1999).

${ }^{21}$ S. C. Erwin, A. A. Baski, L. J. Whitman, and R. E. Rudd, Phys. Rev. Lett. 83, 1818 (1999).

${ }^{22}$ K. Yoo, S. J. Tang, P. T. Sprunger, I. Benito, J. Ortega, F. Flores, P. C. Snijders, M. C. Demeter, and H. H. Weitering, Surf. Sci. 514, 100 (2002).

${ }^{23}$ A. A. Baski and L. J. Whitman, J. Vac. Sci. Technol. B 14, 992 (1996).

${ }^{24}$ O. J. Glembocki and S. M. Proke, Appl. Phys. Lett. 71, 2355 (1997).

${ }^{25}$ A. A. Demkov, J. Ortega, O. F. Sankey, and M. P. Grumbach, Phys. Rev. B 52, 1618 (1995).

${ }^{26}$ O. F. Sankey and D. J. Niklewski, Phys. Rev. B 40, 3979 (1989).

${ }^{27}$ CASTEP 4.2 Academic version, licensed under the UKCP-MSI Agreement, 1999.

${ }^{28}$ N. Mingo, L. Jurczyszyn, F. J. Garcia-Vidal, R. Saiz-Pardo, P. L. de Andres, F. Flores, S. Y. Wu, and W. More, Phys. Rev. B 54, 2225 (1996).

${ }^{29}$ L. Jurczyszyn, J. Ortega, R. Pérez, and F. Flores, Surf. Sci. 482485, 1350 (2001).

${ }^{30}$ J. M. Blanco, C. González, P. Jelínek, J. Ortega, F. Flores, and R. Pérez, Phys. Rev. B 70, 085405 (2004).

${ }^{31}$ J. M. Blanco, C. González, P. Jelínek, J. Ortega, F. Flores, R. Pérez, M. Rose, M. Salmeron, J. Méndez, J. Wintterlin et al., Phys. Rev. B 71, 113402 (2005).

${ }^{32}$ A. A. Baski and L. J. Whitman, Phys. Rev. Lett. 74, 956 (1995). 
${ }^{33}$ S. M. Sze, Physics of Semiconductor Devices (Wiley, New York, 1981).

${ }^{34}$ P. C. Snijders, J. N. Crain, J. L. McChesney, M. C. Gallagher, and F. J. Himpsel (unpublished).

${ }^{35}$ R. M. Feenstra, J. A. Stroscio, and A. P. Fein, Surf. Sci. 181, 295 (1986).

${ }^{36}$ J. Tersoff and D. R. Hamann, Phys. Rev. Lett. 50, 1998 (1983).

${ }^{37}$ J. Tersoff and D. R. Hamann, Phys. Rev. B 31, 805 (1985).

${ }^{38}$ X. Chen, F. Wu, Z. Zhang, and M. G. Lagally, Phys. Rev. Lett. 73, 850 (1994).

${ }^{39}$ P. Gambardella, A. Dallmeyer, K. Maiti, M. C. Malagoli, W. Eberhardt, K. Kern, and C. Carbone, Nature (London) 416, 301
(2002).

${ }^{40}$ Note that the bulk projected gap for the PW (LDA) calculation is not equal to $\sim 0.67 \mathrm{eV}$, as a fully converged PW (LDA) calculation should give. This is caused by the fact that the bands are plotted along symmetry directions in the surface Brillouin zone that do not contain the $k$ point corresponding to the bulk $\mathrm{Si}$ CBM.

${ }^{41}$ The chemical potential of liquid $\mathrm{Ga}$ is approximated by the chemical potential of solid, bulk $\mathrm{Ga}$ at $T=0 \mathrm{~K}$. Finitetemperature corrections which include the enthalpy of melting and the integrated heat capacity are very small $(<0.1 \mathrm{eV})$ and have been neglected. 